SVU- International Journal of Veterinary Sciences, 4 (3): 94-102, 2021.

Print ISSN: 2535-1826

\title{
The effect of CpG-ODN (Toll-like receptor 21 agonist) as an adjuvant for Newcastle disease
} vaccination in broiler chicken

\section{Ghada K. Gamal ${ }^{1}$, Mostafa. A. Shehatta ${ }^{2}$, Shiem M. El-Sherry ${ }^{2 *}$}

${ }^{1}$ Mater student, Department of Avian and Rabbit Diseases, Faculty of Veterinary Medicine, Assiut University, Assiut, Egypt, ${ }^{2}$ Department of Avian and Rabbit Diseases, Faculty of Veterinary Medicine, Assiut University, Assiut, Egypt.

\section{Abstract}

The present study was conducted to evaluate the immune-stimulating effect of CpG-ODN (Toll-like-receptor-agonist21) on broiler chickens vaccinated against Newcastle disease. The motifs were examined with different vaccination programmes using different inoculation routes. 270 (one-day-old) - chicks were divided into 6 groups. Group one as a negative control sham inoculated with PBS; group two was injected intramuscularly with CpG-ODN; group three was vaccinated with live ND vaccine by eye-drop route; group four was vaccinated by eye-drop route and injected with CpG-ODN; group five was vaccinated with Newcastle disease inactivated vaccine and injected intramuscularly with $\mathrm{CpG}-\mathrm{ODN}$; group six was vaccinated by eye-drop route with live attenuated vaccine dissolved in CpG-ODN. Antibodies responses were monitored based on hemagglutination inhibition test and ELISA assay for the detection of IgG. The average body weight gain and feed conversion ratio were estimated to evaluate CpG-ODN growth-promoting effect. All vaccinated groups supplied with CpG-ODN recorded higher antibodies titer than the only vaccinated group. Group 4 which vaccinated and inoculated intra-muscularly with CpG-ODN recorded higher antibodies titer than other vaccinated groups. Antibodies titer was similar in group five and six without significant difference and both were lower than group 4. ELISA assay confirmed the significance between the adjuvanted groups at 21 and 35 days old. There were no significant differences $(\mathrm{p} \geq 0.05)$ observed between the vaccinated and adjuvated groups in either average body weight gain or in feed conversion rate. In conclusion, $\mathrm{CpG}-\mathrm{ODN}$ proved its efficiency as an adjuvant when co-inoculated with Newcastle disease vaccines via different administration routes.

Keywords: Broilers, CpG-ODN, Newcastle disease, Toll like receptors.

DOI: 10.21608/svu.2021.79758.1129 Received: June 8, 2021 Accepted: August 30, 2021 Published: September 30, 2021

*Corresponding Authors Shiem M. El-Sherry

E-mail: shiemelsherry@vet.au.edu.eg Citation: Gamal et al., The effect of CpG-ODN (Toll-like receptor 21 agonist) as an adjuvant for Newcastle disease vaccination in broiler chicken. SVU-IJVS 2021, 4 (3): 94-102.

Copyright: (c) Gamal et al. This is an open access article distributed under the terms of the creative common attribution license, which permits unrestricted use, distribution and reproduction in any medium provided the original author and source are created.

Competing interest: The authors have declared that no competing interest exists. 


\section{Introduction}

Newcastle disease (ND) is considered one of the main constraints that cause huge losses either to backyard rearing or under non ideal farming conditions (Xiao et al., 2009). The virus is a single-stranded RNA (subfamily Avulavirinae) has a persistent nature that leads to a continuous extensive studies aiming to hinder such highly contagious disease. Activating innate and adaptive immune responses play an essential role against ND invasion. The immune response is triggered upon the recognition of pathogen-associated molecular patterns (PAMP) by specific pattern-recognition receptors (PRRs)(De Nardo, 2015). Recently, many discovered elements were proposed as agonists to microorganisms and have the ability to activate or regulate immune response when recognized by PRRs (Steinhagen et al., 2011).

Unmethylated CpG-oligodeoxynucleotides (CpG-ODN) are small molecular motifs conserved in some microorganisms including bacterial and viral DNA. Upon binding to PRRs, they can initiate the release of cytokines and others co-stimulation elements, the process which is essential for triggering rapid innate, and adaptive immune responses (Paul et al., 2013). Therefore, CpG-ODNs were studied for their function as antiviral agents or as vaccine adjuvants. While CpG-ODN proved a strong ability to control viral infection (Dou et al., 2013) and safety for use without necrosis or toxicity induction, interest has increased for using them as vaccinal adjuvants. Proposing CpG-ODN as adjuvants facing several problems including costs, and stability when used under different environmental conditions. In addition to variation in immunemodulation which was recorded due to multiple factors including the nature of microorganism, site of infection, route and dose of inoculation (Gomis et al., 2003; C. Yu et al., 2017).
The present work examined the ability of such adjuvant form to boost humoral immune response generated by ND vaccination as a model for ssRNA virus. The experimental design examined multiple vaccination programs, different inoculating routes and monitoring its effect with either killed or live attenuated vaccines to challenge such adjuvant and to monitor its ability to modulate immune response as well as growth promotion for broiler chickens.

\section{Materials and methods}

\section{Ethical Approval:}

The experimental procedures were carried out in compliance with the institutional bioethics, faculty of veterinary medicine, Assiut University, Egypt.

Birds and Immunization Schedule:

A total of 270 (one-day-old) commercial broiler chicks (Ross) were purchased from (Assiut Poultry Company), weighted and divided into 6 groups (uniformed in the initial body weight) each contained 45 birds ( 3 replicates each $n=15$ ). Group one (G1), as negative control sham inoculated with PBS; group two (G2), injected with CpG-ODN intramuscularly at day 7 and repeated at day 21 for observing the effect of CpG-ODN injection ; group three (G3), vaccinated with live ND vaccine by eye-drop route (Clone 30, international B.V. Boxmeer-Holland) at 7 days old and then with Lasota (international B.V. Boxmeer-Holland) at 21 days old as a control for vaccination; group four (G4), vaccinated by eye-drop route (clone 30 ) at 7 days old plus I/M injection of CpG-ODN and at 21 days old Lasota were given by eye-drop route plus CpG-ODN injection; group five (G5), vaccinated with Newcastle disease inactivated vaccine (Imopest, merial, France) intramuscular injection at the time of vaccination and repeated at 21 days-old; group six (G6), vaccinated with clone 30 dissolved in $\mathrm{CpG}-\mathrm{ODN}$ solution 
by eye-drop at 7 days old and Lasota were given dissolved in CpG-ODN solution at 21 days of age. All groups were randomly allocated into individual compartments at the veterinary hospital, faculty of veterinary medicine, Assiut University. They were raised on litter (wood shaving) and provided with food and water ad libitum. Temperature started with $34^{\circ} \mathrm{C}$ and linearly decreased $2^{\circ} \mathrm{C}$ per week with 24 hours lighting. They were raised on starter ration for the first 14 days with $23 \%$ crude protein and energy of $3000 \mathrm{Kcal} / \mathrm{kg}$ ration and grower ration with $21 \%$ crude protein and $3070 \mathrm{Kcal} / \mathrm{kg}$ ration up to $35 \mathrm{th}$ day of age. All groups except group one received the vaccination program shown in Table 1 against AI, IB and IBD. Chicks were observed daily for any symptoms or mortality for the whole duration of the experiment.

\section{Table 1. Vaccination program of vaccinated experimental groups}

\begin{tabular}{c|c|c}
\hline Vaccines & $\begin{array}{c}\text { Age (day)/route of } \\
\text { administration }\end{array}$ & $\begin{array}{c}\text { Vaccinated } \\
\text { Exp. groups }\end{array}$ \\
\hline $\begin{array}{c}\text { IB vaccine (Zoetis, USA) } \\
\text { ND vaccine (Clone 30, International B.V. } \\
\text { Boxmeer-Holland) }\end{array}$ & $\begin{array}{c}1^{\text {st }} \text { (eyedrops) } \\
7^{\text {th }} \text { (eyedrops) }\end{array}$ & G3, G4 and G6 \\
$\begin{array}{c}\text { ND inactivated vaccine (Imopest, merial) } \\
\text { Avian influenza (H5N1) (MEVAC Co. Egypt) }\end{array}$ & $7^{\text {th } \text { (subcutaneous) }}$ & G5 \\
$\begin{array}{c}\text { Gumboro (CEVAC, France) } \\
\text { Lasota (International B.V. Boxmeer-Holland) }\end{array}$ & $\begin{array}{c}2^{\text {th }} \& 28^{\text {th }} \text { (eyedrops) } \\
21^{\text {st }} \text { (eyedrops) }\end{array}$ & $\begin{array}{c}\text { All groups } \\
\text { G3, G4 and G6 }\end{array}$ \\
\hline
\end{tabular}

\section{Preparation of Vaccine and Adjuvants:}

The CpG-ODN was stored at $-20^{\circ} \mathrm{C}$ until used and diluted in PBS before being administered at a dose of $50 \mu \mathrm{g} /$ chicken as an adjuvant. CPG-ODN was synthesized according to referred sequence (5tcgtcgttgtcgtcgttttgtcgtt-3) (Gomis et al., 2003).

\section{Samples collection:}

Blood samples were weekly collected from all vaccinated and non-vaccinated groups starting from day one up to day 35 of age. Blood samples were collected from chicks (10 birds of each group) either by decapitation at 1 and 7 days of age or were bled via wing vein puncture $(14,21,28$ and 35 days of age). Samples were collected and kept at $37^{\circ} \mathrm{C}$ for $1 \mathrm{hr}$. then centrifuged at $5,000 \mathrm{~g}$ for $30 \mathrm{~min}$; then the serum collected and stored at $-20^{\circ} \mathrm{C}$ to be used later.

\section{Hemagglutination inhibition test:}

The HAU of the NDV was titrated before each assay. ND serum antibodies were quantified by hemagglutination inhibition (HI) assay in U-bottomed 96-well microtiter plates as per the procedure recommended in the OIE manual (Stear, 2005). Briefly, serum samples were twofold diluted.50 ul ND viral antigen was then added to each well. The plate was shaken and incubated for $45 \mathrm{~min}$. $50 \mathrm{uL}$ of $0.5 \%$ chicken red blood cells was added to each well. Plates were shaken and incubated for 45 min. HI titer was expressed as the highest dilution of complete inhibition of agglutination (expressed as $\log 2$ scale).

\section{Enzyme-linked immunosorbent assay:}

To measure the adjuvant ability of $\mathrm{CpG}$ ODN, and to confirm results from $\mathrm{HI}$ test, samples were collected at 21days old and 35days old and tested using ELISA. Briefly, ELISA plates were coated with $100 \mathrm{ul} /$ well of ND antigen $(20 \mathrm{ug} / \mathrm{mL})$ in $50 \mathrm{mM}$ carbonate bicarbonate buffer $(\mathrm{pH} 9.6)$ at 
$4^{\circ} \mathrm{C}$, then washed and blocked per well by $1 \%$ bovine serum albumin (Sigma-Aldrich) and $0.05 \%$ Tween20 in PBS (blocking buffer) was added for a $1 \mathrm{hr}$ incubation period at room temperature. After washing, $100 \mathrm{ul}$ of serum diluted 1/50 was added to each well and incubated for $1 \mathrm{hr}$. Samples were measured induplicate. The plates were washed 3 times then antichicken rabbit anti-chicken IgG antibodies conjugated to horse-radish peroxidase (HRP) was added (Jackson Immuno Research Laboratories, Inc.). The plates were incubated for $1 \mathrm{hr}$. at room temperature. Positive control (positive serum from a previous experiment) and negative controls (phosphate buffered saline) were included for each plate. The plates were washed again and $100 \mathrm{ul}$ of HRP substrate solution TMB (Diagnostic Biochem. Canada Co., Ltd) was added to each well, followed by an incubation period of 15-20 min in the dark at room temperature before the addition of $100 \mathrm{ul}$ of a $1 \%$ stopping solution (Diagnostic Biochem Canada Co., Ltd). The optical density (OD) was determined at $405 \mathrm{~nm}$ using an ELISA plate reader (BioTek Instruments, Winooski, Vermont USA).

\section{Growth performance:}

Chicks were individually weighed at arrival and a weekly (20 birds from each group). Feed intake was also measured at weekly interval during the experiment and feed conversion ratio $(\mathrm{FCR}=$ feed intake /weight gain) were calculated. Weight gain and FCR were calculated for 3 times intervals. Day (1-21), day (21-35) and day (1-35).

\section{Statistical analysis:}

The data for all variables were evaluated by one-way analysis of variance (ANOVA) as a completely randomized design using the Prism software (GraphPad Prism version 8.0.0 for Windows,
GraphPad Software, San Diego, California USA). The means for treatments showing significant differences in the ANOVA were compared using Post-Hoc Tukey and paired-samples t-tests. Differences were considered significant at $\mathrm{p}<0.05$.

\section{Results}

\section{Hemagglutination inhibition test}

The $\log 2 \mathrm{HI}$ titers of all groups at day $14,21,28$, and 35 after vaccination with primary and secondary doses were demonstrated in Fig. 1. At day 14G2 which was injected with $\mathrm{CpG}-\mathrm{ON}$ was higher significantly $(\mathrm{p}<0.05)$ than $\mathrm{G} 1$ with a $\log 2$ $\mathrm{HI}$ titer of 4.75 , the antibodies in $\mathrm{G} 2$ declined similar to $\mathrm{G} 1$ in day21, 28 with a $\log 2 \mathrm{HI}$ titer of 1.12 and 1.63 in day 35 of age without any significant difference. G3 which was vaccinated without $\mathrm{CpG}-\mathrm{ODN}$ had the lowest antibodies titer with a significant difference among the vaccinated groups; G4 ( $\log 2$ 4.37), G5 ( $\log 24)$ and G6 $(\log 24.50)$. At 21 days of age there was a significant difference between G3 with a $\log 2 \mathrm{HI}$ titer of 4.5 and $\mathrm{G} 4$ which injected IM with CpG-ODN $(\log 25.50)$. There was no significant difference between $\mathrm{G} 3(\log 2$ 4.5), G5 and G6 ( $\log 2$ 4.7). G5 was lower significantly than $\mathrm{G} 4$ with a $\log 2 \mathrm{HI}$ titer of 5.5. At day 28, G3 (log2 3.82) was significantly lower than all vaccinated $\mathrm{G} 4$ $(\log 25.50)$, G5 $(\log 25.25)$ and G6 $(\log 2$ 5.25). This significance continued similarly at day 35. G4 recorded the highest level of antibodies titer with a $\log 2 \mathrm{HI}$ titer of 5.50 among the vaccinated G5 $(\log 25.0)$ and G6 ( $\log 2$ 4.7. Antibodies titer increased with a $\log 2 \mathrm{HI}$ titer of 5.50 to become close to G5 $(\log 25.25)$ and G6 $(\log 25.25)$ without significant. These results continued up to day 35. G5. These results continued up to day 35. G6 which was vaccinated with vaccine dissolved in $\mathrm{CpG}-\mathrm{ODN}$ solution showed similar course as G5 without significant difference. 


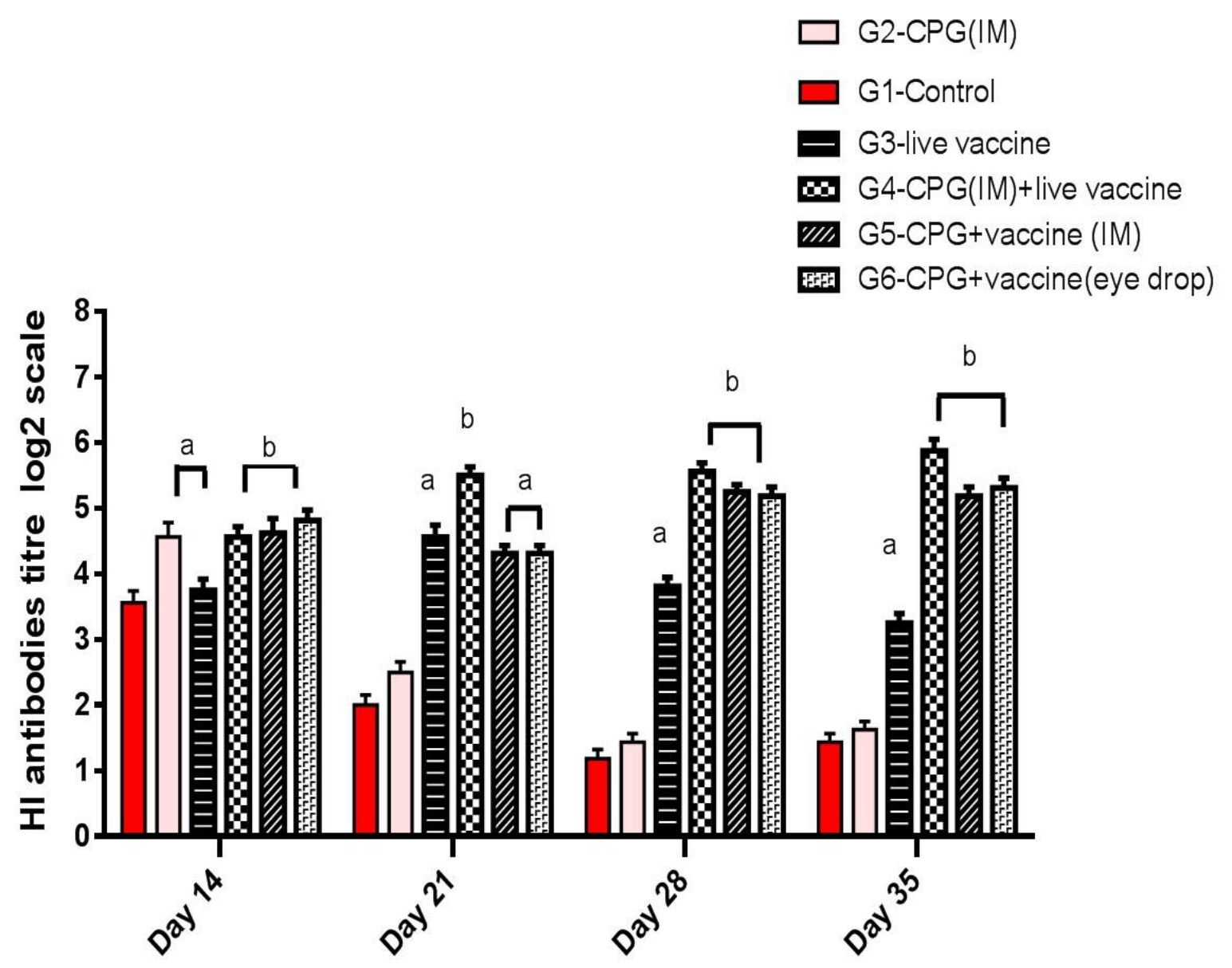

Fig. 1. Newcastle disease HI titers ( $\log 2)$ of experimental groups.

Enzyme-Linked immunosorbent assay:

On day 21, G4 recorded the highest level of antibodies titer $(1467 \pm 13.72)$ among the vaccinated G3 (554 \pm 11.02$)$, G5

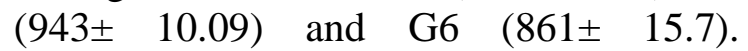
Antibodies titer was similar in G5 and G6 without significance. A significant difference was observed between G3 (554 \pm $11.02)$ and $G 2$ (312 \pm 11.54$)$. No significant difference was observed between G3, G5 and G6. At 35 day of age, G4 (3124 \pm 80.33$)$ recorded the highest level of antibodies titer (3124 \pm 80.33) among the vaccinated G3 (1329 \pm 38.65$),$ G5 (1745 \pm 38.23$)$ and G6 (1738 \pm 81.30) (Fig. 2.). Antibodies titer was similar in G5 and G6 without significant.

Growth performance
The comparison between all the experimental groups at day $0,7,14,21,28$, and 35 was shown in Fig. 3. and Table 2. There were no significant differences $(p \geq 0.05)$ were observed between different vaccinated and adjuvanted groups in either average body weight gain (g) or in feed conversion rate starting from day 7 of age up to the end of the experiment. The gain of vaccinated groups had no significant difference $(p \geq 0.05)$ in comparison with negative control chicken. The injection of CpG-ODN did not affect body weight gain or FCR whether inoculated alone $(\mathrm{G} 2)$ or with vaccines using different inoculation routes (G4, G5 and G6). Although (G4) which was vaccinated by eye-drop route and injected IM with $\mathrm{CpG}-\mathrm{ODN}$ recorded the highest weight gain among the vaccinated groups, no significant difference was found up to the end of the experiment. 


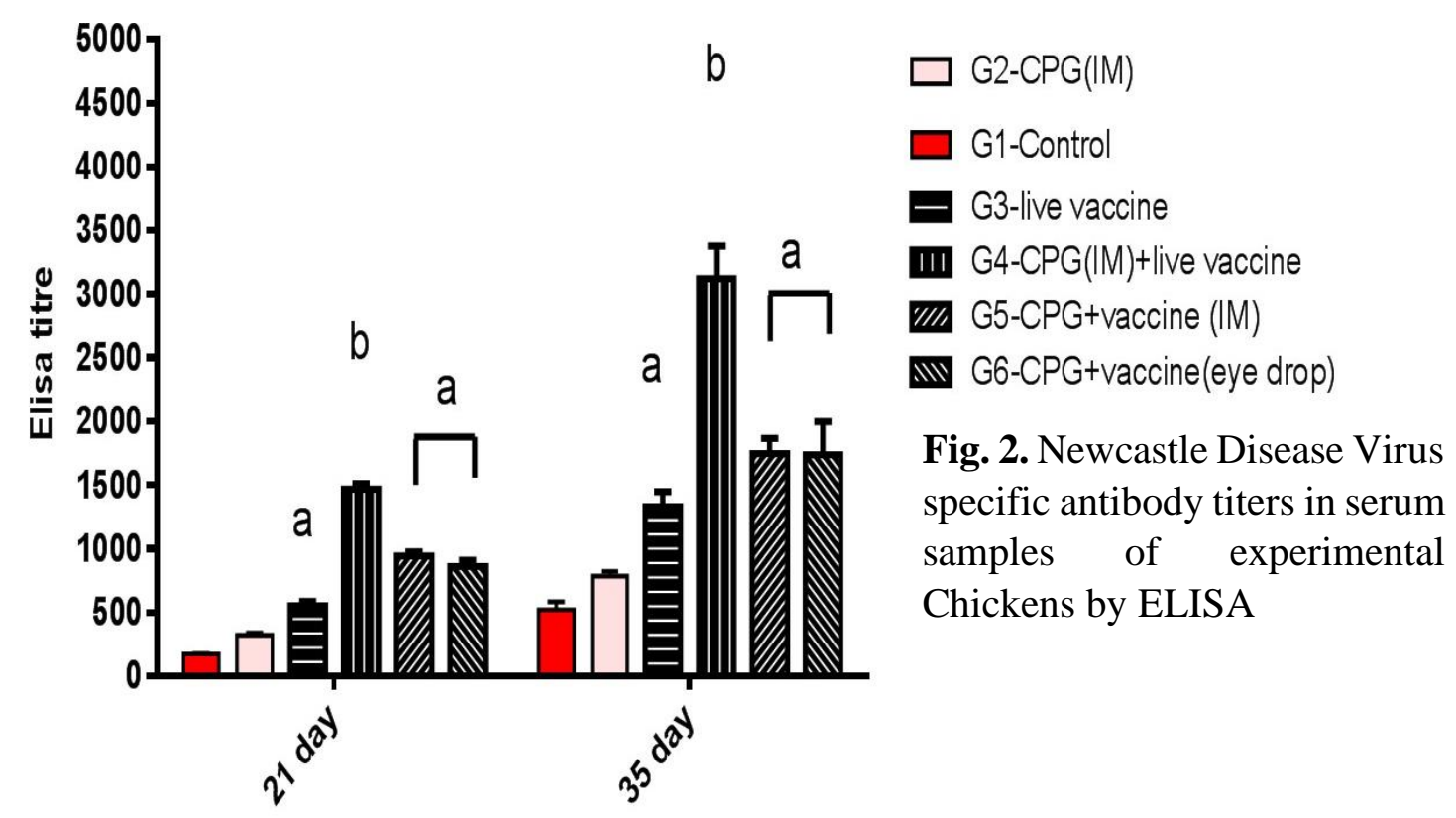

Table 2. Growth performance of different experimental groups

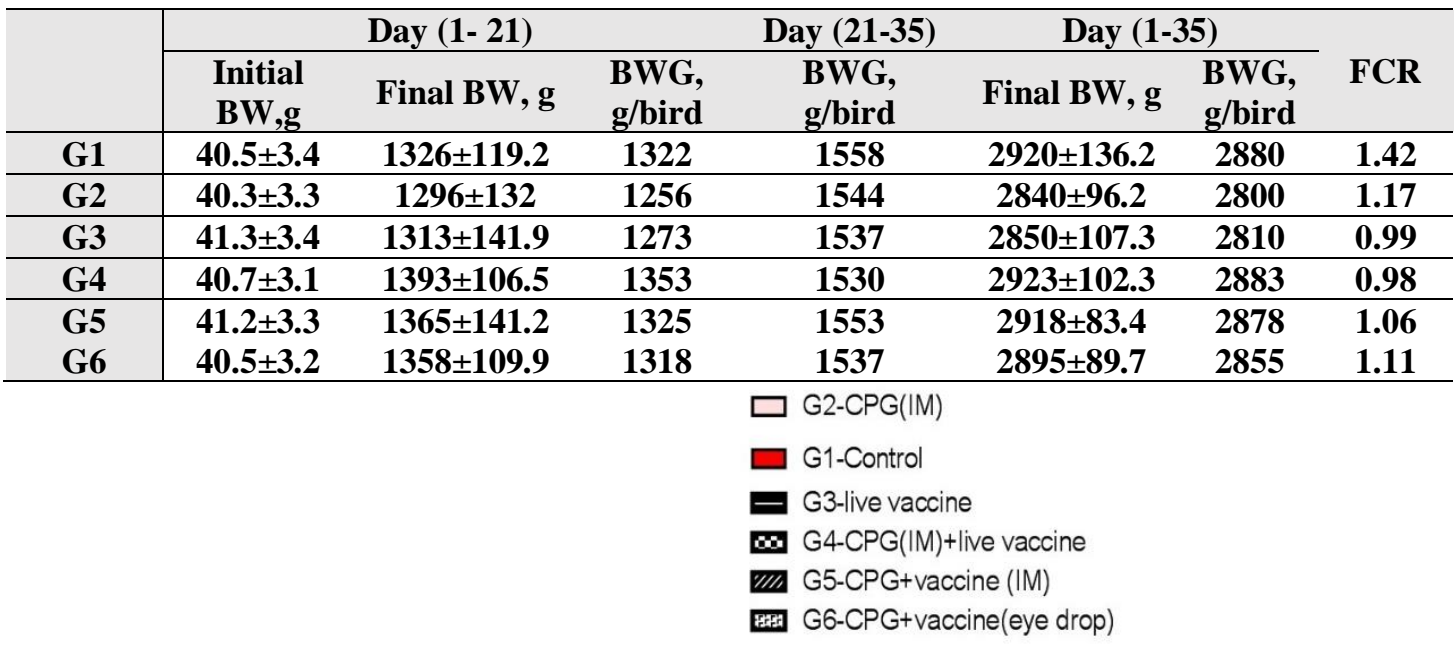

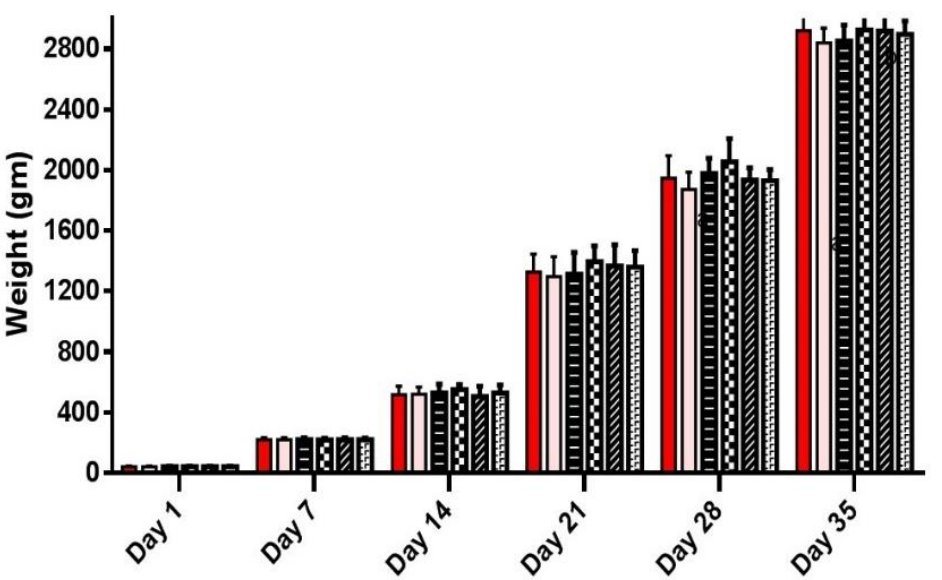

Fig. 3. Growth performance of different experimental groups

\section{Discussion}

Poultry flocks which vaccinated with ND vaccine are not fully shielded from the NDV infection, even with a considerable antibodies level induced by vaccination. The ability of TLR agonists to affect immune response in chicken is now reported in many studies (Chen and Wang, 2013). It is well known that many adjuvants such as 
aluminium gel initiate mainly humoral immune response rather than cell-mediated (HogenEsch, 2002). CpG motifs can trigger innate, cellular and humoral responses. Moreover, the CpG-ODN initiate stronger antibodies production than common adjuvants such as Freund's. In addition, Freund's frequently lead to undesirable side effects such as abscesses and inflammation at the inoculation site. Therefore, $\mathrm{CpG}$ ODN is proposed as a strong potential treatment or more efficient vaccine adjuvant. However, scarce data are available regarding using these motifs in a form that can be used with commercial chicken and produce consistent results. When inoculating with vaccines, CpG-ODN can initiate B-lymphocytes activation, and differentiation of the plasma cell which leads to increase ND-specific antibodies release. In the present work, all vaccinated groups supplied with $\mathrm{CpG-ODN}$ recorded higher antibodies titre than only the vaccinated group started from 14 days postimmunization. G4 which vaccinate and inoculated IM with CpG-ODN recorded higher antibodies titre than other vaccinated groups. CpG-ODN is used via different routes including oral, intranasal, subcutaneous and intramuscular. Different methods of inoculation of CpG-ODN can affect its capability of immune response modulation (Chrząstek et al., 2014). It is recorded in several studies that $\mathrm{CpG}$ ODN given parenterally induces strong Th1 components like IL-2, IL-12, IFN-c, IgG2a (Yi et al., 1996). IFN-c is an important B cell key for the promoting of ND IgG2a secreting B cells (Wang et al., 2009).As the administration of the CpG-ODN motifs in the antigen entrance sites was advised as an important factor for its adjuvating efficiency (B. Yu et al., 2017), no significant difference between adjuvanted groups were observed at the end of the experiment. indicating that routes of vaccination and administration of $\mathrm{CpG}$ did not effect on immune responses as expected. The three adjuvanted groups were not significantly different from only the vaccinated group up to 21 days of age except group 4 which was higher than all vaccinated groups. This observation can be explained by previous observation which proposed that intraocular application of the CpG ODN did not trigger the same response, as when administered parenterally. The reason might be that the $\mathrm{CpG}$ ODN reacted with different cells on the inoculation site. After subcutaneous or intramuscular administration, the ODNs are reacted with antigen-presenting cells, dendritic cells and macrophages(Akiba et al., 2004). While eyedrop application encounter mainly with the Harderian gland (B cells, plasmatic cells, $\mathrm{T}$ cells and scares macrophages) (Śmiałek et al., 2011).At day 28,35 and 42 days of age, antibodies titer of all groups were close although of different inoculation routes that were used in the second immunization. While intramuscular co-injection of $\mathrm{CpG}$ ODN induces more potent immune responses (serum IgG titer and cellular proliferation) (Linghua et al., 2007)that results in further enhancement of immune responses above levels achieved by the effect of common adjuvants. It is reported that local mucosal application of CpG-ODN stimulated both T-helper IgG and $\operatorname{IgA}$ (Zhang et al., 2008). CpG-ODN has proven a great ability to enhance the systemic antibody of $\operatorname{IgG}$ subtype predominantly transudate into the respiratory tract and afford long-lived protection (Kreijtz et al., 2011). Based on the specific IgG ELISA, there was obvious increase in the titre of specific antibodies at 21 days of age with the highest titre induced by IM adjuvated group 4. G4 were higher significantly than G5 (inactivated vaccine+ intraocular CPGODN) and G6 (vaccine dissolved in CpGODN ND given intraocular) at day 21 (14 days after first immunization) and peaked up at day 35 of age (14 days after second immunization). The effect of a single treatment with CpG-ODN was recorded to last for about up to 9 days, but high level of antibodies can be maintained for longer time by re-administration of CpG-ODN (Barri, 2005). G2 which injected with CpG-ODN 
slow down the MDA decline up 14 days of age in comparison to G1. The effect of the CpG-ODN on the persistent time of antibodies was reported before (Vleugels et al., 2002). They reported that antibodies in CpG-ODN inoculated group declined slower than control group. In conclusion, the present study showed that inoculation of $\mathrm{CpG}$ was able to elicit higher serum antibody responses compared to vaccinated only groups with different inoculation route. More studies may be conducted to establish a more stable form of these motifs or included them in the vaccine genetic structure to get the most beneficial effect of its immunostimulant properties.

\section{Conflict of interests}

All authors declare that there is no conflict of interest.

\section{Financial disclosures}

This work was not supported by any financial support.

\section{References}

Akiba H, Satoh M, Iwatsuki K, Kaiserlian D, Nicolas J-F, and Kaneko, F (2004). $\mathrm{CpG}$ immunostimulatory sequences enhance contact hypersensitivity responses in mice. Journal of Investigative Dermatology, 123(3): 488-493.

Barri, A (2005). Effects of Cytosinephosphate-Guanosine Oligo deoxy nucleotides (CpG-ODN) on vaccination and immunization of neonatal chickens. Texas A\&M University.

Chen S, Cheng A, and Wang M (2013). Innate sensing of viruses by pattern recognition receptors in birds. Veterinary Research, 44(1): 1-12.

Chrząstek K, Borowska D, Kaiser P, and Vervelde L (2014). Class B CpG ODN stimulation upregulates expression of TLR21 and IFN- $\gamma$ in chicken Harderian gland cells. Veterinary Immunology and Immunopathology, 160(3-4): 293-299.

de Nardo D (2015). Toll-like receptors: activation, signalling and transcriptional modulation. Cytokine, 74(2): 181-189.

Dou W, Li H, Cheng Z, Zhao P, Liu J, Guo H (2013). Maternal antibody induced by recombinant gp 85 protein vaccine adjuvanted with CpG-ODN protects against ALV-J early infection in chickens. Vaccine, 31(51): 61446149.

Gomis S, Babiuk L, Godson DL, Allan B, Thrush T, Potter A (2003). Protection of chickens against Escherichia coli infections by DNA containing $\mathrm{CpG}$ motifs. Infection and Immunity, 71(2): 857-863.

HogenEsch H (2002). Mechanisms of stimulation of the immune response by aluminum adjuvants. Vaccine, 20: S34-S39.

Kreijtz J, Fouchier RA, Mand Rimmelzwaan GF (2011). Immune responses to influenza virus infection. Virus Research, 162(1-2): 19-30.

Linghua Z, Xingshan T, and Fengzhen Z (2007). Vaccination with Newcastle disease vaccine and $\mathrm{CpG}$ oligodeoxynucleotides induces specific immunity and protection against Newcastle disease virus in SPF chicken. Veterinary Immunology and Immunopathology, 115(3-4): 216222.

Paul MS, Brisbin JT, Abdul-Careem MF, and Sharif S (2013). Immunostimulatory properties of Tolllike receptor ligands in chickens. Veterinary Immunology and Immunopathology, 152(3-4): 191- 
199.

Śmiałek M, Tykałowski B, Stenzel T, and Koncicki A (2011). Local immunity of the respiratory mucosal system in chickens and turkeys. Polish Journal of Veterinary Sciences.

Stear MJ (2005). OIE Manual of Diagnostic Tests and Vaccines for Terrestrial Animals (Mammals, Birds and Bees) 5th Edn. Volumes 1 \& 2. World Organization for Animal Health 2004. ISBN $929044 \quad 622$ 6.€ 140. Parasitology, 130(6): 727.

Steinhagen F, Kinjo T, Bode C, and Klinman DM (2011). TLR-based immune adjuvants. Vaccine, 29(17): 3341-3355.

Vleugels B, Ververken C, and Goddeeris BM (2002). Stimulatory effect of $\mathrm{CpG}$ sequences on humoral response in chickens. Poultry Science, 81(9): 1317-1321.

Wang Y, Shan C, Ming S, Liu Y, Du Y, and Jiang G (2009). Immunoadjuvant effects of bacterial genomic DNA and $\mathrm{CpG}$ oligodeoxynucleotides on avian influenza virus subtype $\mathrm{H} 5 \mathrm{~N} 1$ inactivated oil emulsion vaccine in chicken. Research in Veterinary Science, 86(3): 399-405.

Xiao C, Bao G, and $\mathrm{Hu}$ S (2009).
Enhancement of immune responses to Newcastle disease vaccine by a supplement of extract of Momordica cochinchinensis (Lour.) Spreng. seeds. Poultry Science, 88(11): 2293-2297.

Yi A-K, Chace JH, Cowdery JS, and Krieg AM (1996). IFN-gamma promotes IL6 and $\operatorname{IgM}$ secretion in response to $\mathrm{CpG}$ motifs in bacterial DNA and oligodeoxynucleotides. The Journal of Immunology, 156(2): 558-564.

Yu B, Dong X, Gravina S, Kartal Ö, Schimmel T, Vijg J (2017). Genomewide, single-cell DNA methylomics reveals increased non-CpG methylation during human oocyte maturation. Stem Cell Reports, 9(1): 397-407.

Yu C, An M, Li M, and Liu H (2017). Immunostimulatory properties of lipid modified $\mathrm{CpG}$ oligonucleotides. Molecular Pharmaceutics, 14(8): 2815-2823.

Zhang L, Zhang M, Li J, Cao T, Tian X, and Zhou F (2008). Enhancement of mucosal immune responses by intranasal co-delivery of Newcastle disease vaccine plus $\mathrm{CpG}$ oligonucleotide in SPF chickens in vivo. Research in Veterinary Science, 85(3): 495-502. 